\title{
The analysis of the broad hydrogen Balmer line ratios: Possible implications for the physical properties of the broad line region of AGNs
}

\author{
D. Ilić ${ }^{1,2}$, L. Č. Popović ${ }^{3,2}$, G. La Mura ${ }^{4}$, S. Ciroi ${ }^{4}$, and P. Rafanelli ${ }^{4}$ \\ ${ }^{1}$ Department of Astronomy, Faculty of Mathematics, University of Belgrade, Studentski trg 16, 11000 Belgrade, Serbia \\ e-mail: dilic@matf.bg.ac.rs \\ 2 Isaac Newton Institute of Chile, Yugoslavia Branch, 11060 Belgrade, Serbia \\ 3 Astronomical Observatory, Volgina 7, 11160 Belgrade, Serbia \\ ${ }^{4}$ Dipartimento di Fisica e Astronomia, Università di Padova, Vicolo dell’Osservatorio, 35122 Padova, Italy
}

Received 28 March 2012 / Accepted 10 May 2012

\begin{abstract}
Aims. We analyze the ratios of the broad hydrogen Balmer emission lines (from $\mathrm{H} \alpha$ to $\mathrm{H} \varepsilon$ ) in the context of estimating the physical conditions in the broad line region (BLR) of active galactic nuclei (AGNs).

Methods. Our measurements of the ratios of the Balmer emission lines are obtained in three ways: i) using photoionization models obtained with a spectral synthesis code CLOUDY; ii) applying the recombination theory for hydrogenic ions; iii) measuring the lines in observed spectra taken from the Sloan Digital Sky Survey database. We investigate the Balmer line ratios in the framework of the so-called Boltzmann plot (BP), analyzing the physical conditions of the emitting plasma for which we could use the BP method. The BP considers the ratio of Balmer lines normalized to the atomic data of the corresponding line transition, and in that way differs from the Balmer decrement.

Results. We find that for a certain range of thermodynamic parameters, there are objects that follow the BP. These AGNs may have a BLR consisting of mostly high density plasma.
\end{abstract}

Key words. galaxies: active - quasars: emission lines - line: formation - plasmas

\section{Introduction}

It remains important to study the broad line region (BLR) structure (size, geometry, physics, etc.) of active galactic nuclei (AGNs). One of the main applications of the BLR investigations is to accurately estimate the mass of the super-massive black hole (SMBH) in the center of an AGN, which can be derived from the dynamics of the BLR gas that is gravitationally bound to the SMBH (see e.g. McGill et al. 2008; Bentz et al. 2010). After several decades of research, our understanding of the physics and structure of the BLR continues to improve, but there are still many remaining uncertainties in its properties (see e.g. Baldwin 1997; Sulentic et al. 2000; Gaskell 2009, for a review). It is obvious from the broad emission line (BEL) fluxes and profiles that the BLR physics and kinematics are more complicated than in the narrow line region (NLR) or other nebular environments (see e.g. Krolik 1999; Sulentic et al. 2000; Osterbrock \& Ferland 2006, and references therein), and they are even closer to the conditions in stellar atmospheres than in gaseous nebulae (Osterbrock \& Ferland 2006).

Most of the BLR gas is photoionized. This was first inferred from the approximately constant value of the $\mathrm{H} \beta$ equivalent width in several AGNs (Osterbrock 1978) and then confirmed by reverberation studies showing a direct response of the BEL fluxes to the continuum flux changes (see e.g. Peterson 1994). There have been numerous attempts to describe the BLR physics and explain the emission-line spectrum in terms of photoionization models (see e.g. Netzer 1975; Davidson \& Netzer 1979; Kwan \& Krolik 1981; Collin-Souffrin et al. 1982; Rees et al. 1989; Baldwin et al. 1995, 1996; Dumont et al. 1998; Ferland et al. 1998; Krolik 1999; Marziani et al. 2001; Ferland 2003; Korista \& Goad 2004; Leighly \& Casebeer 2007; Snedden \& Gaskell 2007; Marziani et al. 2010). Today, there are roughly half a dozen major codes, that have been continuously developed since the 1970s (see e.g. Ferland \& Savin 2001, for a review). Although these numerical simulations have largely improved in the course of the years, the investigation of the BLR can still help to resolve some important outstanding problems (see e.g. Ferland 1999). One of the most interesting concerns the observed small ratio among the $\operatorname{Ly} \alpha$ and $\mathrm{H} \beta$ lines (which is usually in the range of 5-15), while models predict much higher values (30-50) (see e.g. Netzer et al. 1995, and references therein). Another important finding is that there are indications for a physical complexity of the BLR, which could result from the combination of distinct components, as suggested, for instance, by the origin of "high" and "low ionization lines" (see e.g. Collin-Souffrin \& Lasota 1988; Baldwin 1997; Sulentic et al. 2000), but also according to the complex profiles of single lines, which cannot be explained in terms of a simple singleregion model (see e.g. Popović et al. 2004; Popović 2006b; Bon et al. 2009; Gaskell 2009).

Different BELs originate at various distances from the central continuum source and under a wide range of physical conditions (see e.g. Sulentic et al. 2000). The BLR has a high 
density, thus the collisional excitation, self-absorption, dust obscuration, and complicated coupling of line and continuum radiation transfer should be taken into account in the calculation of the resulting spectrum (see e.g. Baldwin 1997). In these circumstances, the general spectroscopic techniques, which are commonly adopted to estimate the physical conditions in nebular environments, cannot be applied. Some clues, such as the observed Fe II emission, suggest that $T \leq 35000 \mathrm{~K}$, since at higher temperatures it would be effectively suppressed by collisional ionization to Fe III (Osterbrock \& Ferland 2006). However it is very likely that the Fe II emission only affects a fraction of the BLR (see e.g. Marziani et al. 2001; Ilić et al. 2009; Popović et al. 2009; Kovačević et al. 2010, and references therein). The electron density is estimated to be in the range $10^{8} \mathrm{~cm}^{-3} \lesssim n_{\mathrm{e}} \lesssim 10^{14} \mathrm{~cm}^{-3}$, where this range is defined by the need to suppress the emission of broad forbidden lines, while still allowing for the presence of permitted and semi-forbidden ones (Osterbrock \& Ferland 2006). For example, for the upper limit the CIII] $\lambda 1909$ emission line implies that the densities cannot be higher than $\sim 10^{11-13} \mathrm{~cm}^{-3}$, though strong ultraviolet (UV) lines such as Fe II or Al III $\lambda 1860$ suggest somewhat higher density, at least in the low ionization region (Baldwin et al. 1996; Laor et al. 1997; Negrete et al. 2011).

Not many proposed methods in the literature include observations of the BELs to determine the BLR physical properties (Marziani et al. 2001; Laor 2006). Marziani et al. (2001) found, using the CLOUDY photoionization computation, that the ratio of Si III] $\lambda 1892$ to C III] $\lambda 1909$ UV emission lines is a good density diagnostic in the density range $9.5 \lesssim \log n_{\mathrm{e}} \lesssim 12$. Since this ratio is correlated with the width of the broad $\mathrm{H} \beta$ line, these authors presented a relationship for the estimates of the electron density in the BLR using either the ratio of these semi-forbidden lines or the width of the broad component of the $\mathrm{H} \beta$ line (Marziani et al. 2001). The same authors extended their analysis and analyzed the physical conditions using other line ratios (e.g. Al III $\lambda 1860 / \mathrm{Si}$ III] $\lambda 1892$, Si III] $\lambda 1892 / C$ IV $\lambda 1549$, $\mathrm{Mg}$ II $\lambda 2800 / \mathrm{Ly} \alpha$, etc.) trying to exploit the CLOUDY simulations to deduce constraints on the ionization parameter, density, and column density (Marziani et al. 2010). In addition, Laor (2006) proposed a method that considers the electron scattering influence on the line profiles and determines the physical parameters of the BLR in the case of low-luminosity AGNs with emission-line profiles having exponential wings (e.g. the case of galaxy NGC 4395). The method assumes that the exponential wings are produced by the optically thin, isotropic, thermal electron scattering. In this case, by fitting the line wings with an electron-scattering model, one can estimate the electron density and optical depth of the region (Laor 2006). Both described methods are observationally constrained using either UV observations or detecting BEL with strong exponential line wings to extract a direct estimate.

On the other hand, Popović et al. (2002) and Popović (2003, 2006a) suggested that the Boltzmann-plot (BP) method (see Sect. 2.2 for more details), a previously developed and well-known laboratory diagnostic of high density plasma (Griem 1997), might be exploited to probe the BLR of some AGNs (see also Ilić et al. 2006; La Mura et al. 2007; Popović et al. 2008). By measuring the flux of emission lines belonging to a specific transition series, such as the hydrogen Balmer line series in the optical domain (Popović 2003), and using the corresponding atomic parameters, the technique provides an estimate of the plasma temperature. For example, in the case of NGC 5548 the BLR temperature was estimated using the BP applied to the Balmer lines observed from 1996 to 2004 (Popović et al. 2008).
A strong correlation between the variation in the optical AGN continuum and the BP temperature was found (Popović et al. 2008).

The main motivation of this paper is to investigate under which particular circumstances the Balmer line ratios and BP method may, or may not, be used to explore the physical conditions of the BLR, given that photoionization is expected to control the plasma physical properties. To clarify this, we compare the hydrogen Balmer lines (from $\mathrm{H} \alpha$ up to $\mathrm{H} \varepsilon$ ) that we obtained in three different ways: i) from a grid of several numerical models, computed using the CLOUDY spectral synthesis code (Ferland et al. 1998) and analyzing the properties of the resulting spectra; ii) by considering the calculated emission line intensities predicted in the framework of the recombination theory (Storey \& Hummer 1995a), and iii) by analyzing a set of observed spectra taken from the Sloan Digital Sky Survey (SDSS) database (La Mura et al. 2007).

The paper is organized as follows: in Sect. 2, we present the use of the Balmer line ratios in the Balmer decrement and BP method; in Sect. 3, we describe the grids of models generated with the CLOUDY code and report the results of the models, and we describe and analyze the theoretical recombination emission lines; in Sect. 4, we report on the observed SDSS spectra; in Sect. 5, we provide discussion and finally, in Sect. 6 we give our conclusions.

\section{The ratios of the hydrogen Balmer lines - indications of the BLR physical properties}

The hydrogen Balmer lines are the brightest recombination lines in the optical spectra of AGNs. They usually have complex line profiles, composed of narrow and broad components. Here we discuss only the broad component, originating in the BLR.

\subsection{The Balmer decrement}

The hydrogen Balmer decrement - $\mathrm{H} \alpha / \mathrm{H} \beta$ - ratio can be used to probe the physics of the line-emitting plasma (Kwan \& Krolik 1979, 1981; Mathews et al. 1980; Canfield \& Puetter 1981; Dong et al. 2008; Jin et al. 2012). It is most frequently used to determine the amount of dust extinction for the low-density gas, such as the narrow line region (NLR) in AGNs (Osterbrock \& Ferland 2006), where a value of 3.1 for $\mathrm{H} \alpha / \mathrm{H} \beta$ ratio is generally adopted. This is slightly higher than the standard case B recombination value as the $\mathrm{H} \alpha$ emission is enhanced due to collisional effects and harder ionizing continuum (Gaskell \& Ferland 1984; Osterbrock \& Ferland 2006).

The densities in the BLR are far higher, as discussed above, so that the $\mathrm{H} \alpha / \mathrm{H} \beta$ ratio might be affected by high-density effects or wavelength-dependent extinction by dust (see e.g. Osterbrock 1984; Goodrich 1995). The observed broad-line $\mathrm{H} \alpha / \mathrm{H} \beta$ ratio of most broad-line AGNs is usually larger (steeper) than the Case B recombination value (see e.g. Osterbrock 1977; Rafanelli 1985; Dong et al. 2005), sometimes even as steep as ten or higher (Osterbrock 1981; Crenshaw et al. 1988). Dong et al. (2008) obtained the average value of the broad-line Balmer decrement of 3.06, for a large, homogeneous sample of 446 low-redshift $(z \leq 0.35)$ blue type 1 AGNs with the minimal dust extinction effects. They argue that the broad-line $\mathrm{H} \alpha / \mathrm{H} \beta$ ratio can be used as dust extinction estimator, even for the BLR, especially for radio-quiet AGNs (Dong et al. 2008).

On the other hand, in several monitoring campaigns, the Balmer decrement is found to vary, usually anti-correlated with 
the continuum flux, in a single object (Shapovalova et al. 2004, 2010; Popović et al. 2011), but often exhibiting complicated behavior depending on the state of the activity in the AGNs. In the case of NGC 4151, it varied from 2 to 8 during 11 years (Shapovalova et al. 2008), usually decreasing when the continuum increases, but staying constant in the case of high continuum flux. A similar effect was detected in the case of 3C 390.3: for the low activity period, the Balmer decrement anti-correlates with the continuum, while in the outbursting phase with higher values of the continuum flux, it stays almost constant at the value of $\sim 4.5$ (Popović et al. 2011). Thus, the changes in the Balmer decrement might indicate that sometimes the line production is not fully governed by the input ionizing continuum coming from the accretion disk, i.e., the main cause of the Balmer decrement variations is unrelated to the active nucleus and the shock initiated emission is probably dominant (Shapovalova et al. 2010). Or in addition to the physical conditions across the emitting region or input ionizing flux, the size of the emitting regions could play an important role (Popović et al. 2011). For the intrinsic host galaxy reddening in these galaxies, we might expect that during the considered time interval, the intrinsic reddening can be neglected, because it should not vary too much for such a relatively short period ( $\sim 10$ years). The effects of dust within the BLR might still of course, be present.

As we noted above, the Balmer decrement (only the $\mathrm{H} \alpha / \mathrm{H} \beta$ ratio) may be an important diagnostics for some physical processes in the BLR, but it may be more reliable to take into account the ratios of more lines from the Balmer series. One way of readily considering the ratios of several lines is to use the Boltzmann plot. We now provide a short outline of the BP method in Sect. 2.2.

\subsection{The Boltzmann plot}

If a plasma extending over a region of length $\ell$ emits along the line of sight, assuming that the temperature and emitter density do not vary too considerably, the flux $F_{u l}$ of a transition from an upper level $u$ to the lower $l$ can be calculated as (Griem 1997; Konjević 1999; Popović 2003, 2006a; Popović et al. 2008)

$F_{u l}=\frac{h c}{\lambda} g_{u} A_{u l} \frac{N_{0}}{Z} \exp \left(-E_{u} / k T\right) \ell$

where $\lambda$ is the transition wavelength, $g_{u}$ is the statistical weight of the upper level, $A_{u l}$ is transition probability, $N_{0}$ is the average total number density of radiating species that effectively contribute to the line flux without being absorbed, $Z$ is the partition function, $E_{u}$ is the energy of the upper level, $T$ is the average excitation temperature, and $h, c$, and $k$ are the Planck constant, the speed of light, and the Boltzmann constant, respectively. Equation (1) also assumes that the occupation number of the upper level in the transition follows the Saha-Boltzmann distribution, the lines are optically thin, and the line series originates in the same emitting region (for a more detailed derivation and discussion on these assumptions see, Popović 2003, 2006b; Popović et al. 2008).

Introducing a "normalized" line-flux $F_{\mathrm{n}}$, with respect to the atomic constants that characterize the transition

$F_{\mathrm{n}}=\frac{F_{u l} \cdot \lambda}{g_{u} A_{u l}}$

we see from Eq. (1) that it is possible to write

$\log _{10}\left(F_{\mathrm{n}}\right)=B-A E_{u}$, where, considering lines that belong to a specific transition series, $B$ and $A$ represent the Boltzmann-plot parameters, with $B$ being a characteristic constant of the transition series and $A=\log _{10}(e) / k T_{\text {exc }} \approx 5040 / T_{\text {exc }}$ being called the temperature parameter.

Therefore, taking into account a specific line series, such as the Balmer lines, if the populations of the upper energy states $(n \geq 3)^{1}$ follow a Saha-Boltzmann distribution, Eq. (3) provides an estimate of the average excitation temperature in the region where the lines are formed. In the case of the narrow lines which originate in a low density environment, the above assumptions do not apply (see Popović 2003), but for higher densities in the BLR plasma, the approximation might be reasonable. The Balmer line ratios are less sensitive to temperature changes at higher temperatures (Griem 1997). For further analysis, we adopt the maximal value of the BP temperature to be $30000 \mathrm{~K}$, as the BLR temperature should be lower than that (Osterbrock \& Ferland 2006).

The Boltzmann plot should not be confused with the Balmer decrement, as it considers a line ratio normalized using the atomic parameters of the specific line transitions. However, all the discussion presented for the Balmer decrement in the previous section, applies to the BP since the line ratios could be highly influenced by the dust effects. On the other hand, the BP method has the clear advantage of requiring only the measured Balmer line fluxes to estimate the excitation temperature. However, one has to consider some possible drawbacks of the use of emission lines to infer the BLR physical properties, which in general appear in all methods that use BEL parameters for plasma diagnostics. Since the BEL profiles are complex, the estimate of their fluxes should deal with the possibility of a multiplecomponent structure in the BLR, as stated in previous sections. Furthermore, there are some indications that the Balmer lines do not necessarily have to arise strictly from the same region, as it is demonstrated by some differences in the profiles of $\mathrm{H} \alpha$ and $\mathrm{H} \beta$ (see e.g Shapovalova et al. 2008; Popović et al. 2011). Finally, different mechanisms may contribute to the formation of the emission lines and, though photoionization is the main heating source, other processes might be relevant. The combination of these effects implies that the actual properties of the BLR may or may not fit in the fundamental assumptions of the BP method, as illustrated in the examples plotted in Fig. 1.

\section{Simulated and theoretical Balmer line ratios}

\subsection{Simulation of photoionized region and modeled Balmer lines}

Numerical simulations make it possible to understand complex physical environments starting from fundamental principles of physics. This is particularly applicable to the BLR where owing to different physical conditions in the plasma (especially high density), many physical processes should be taken into account. To model this complex physical scenario, our choice was to use CLOUDY ${ }^{2}$ (Ferland et al. 1998). Collisional effects, including excitation and de-excitation, continuum fluorescence, recombination, etc., are all accounted for in this code (Ferland et al. 1998; Ferland 2006).

To investigate the Balmer lines, we generate grids of photoionization models of the BLR using the version C08.00

\footnotetext{
${ }^{1}$ Since the emission de-excitation goes as $u \rightarrow l$, level $l$ needs not to have a Saha-Boltzmann distribution.

2 All details related to this code can be found at:

http://www . nublado.org/
} 

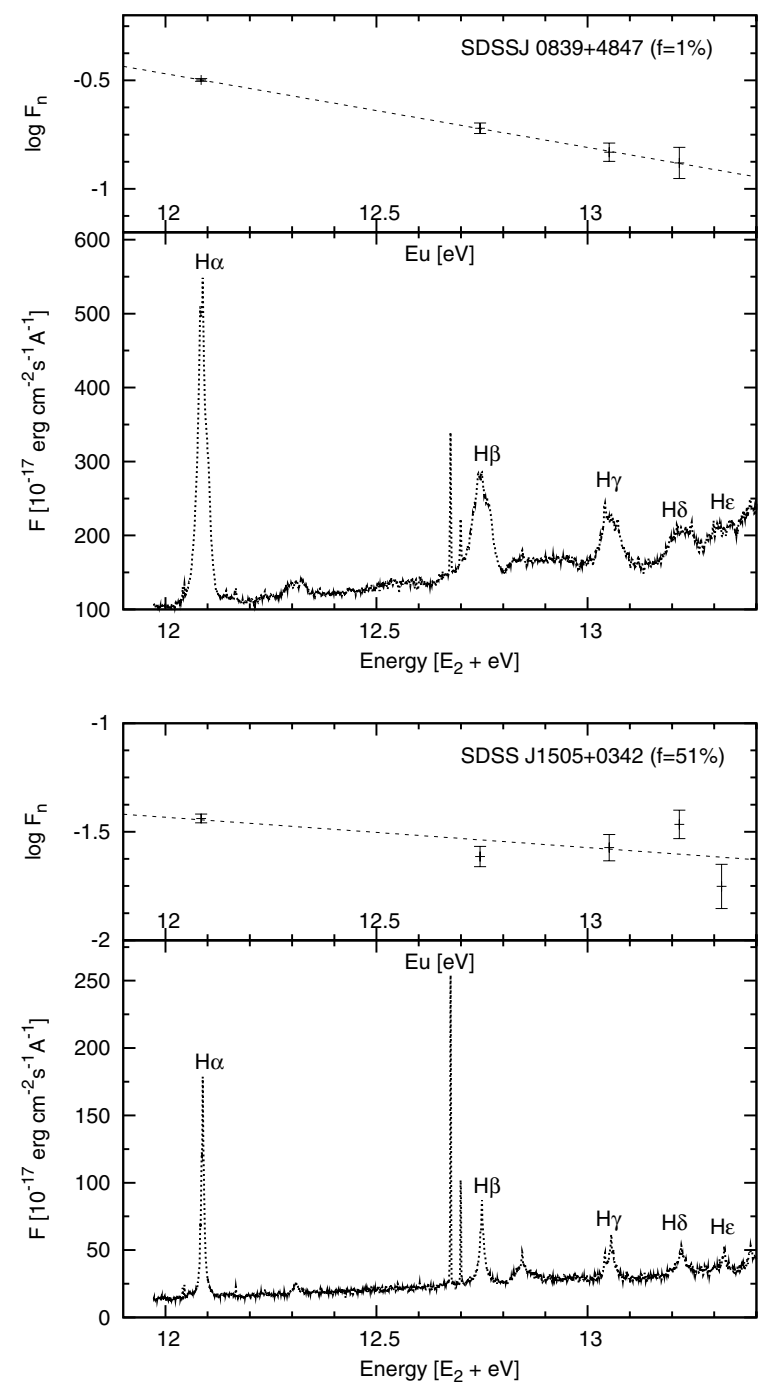

Fig. 1. Examples of AGNs where the BP method works (upper panel) and where it does not give valid results (bottom panel) applied to the data taken from La Mura et al. (2007). Each panel contains the observed spectrum and the BP applied to the line fluxes of the Balmer series (only the broad component of the Balmer lines was used). For the lower plots, which give the observed spectra, the observed wavelength scale ( $x$-axis) is converted into energy units $(\mathrm{eV})$, according to the energy above the ground of the upper transition levels, to match the BP in the upper plots.

of CLOUDY (Ferland et al. 1998; Ferland 2006). This version is roughly a factor of two slower than C07.02, owing to a major improvement in the physics of the $\mathrm{H}$-like iso-sequence. The $l$-levels of the H-like sequence are now fully resolved, making these atoms formally correct, thus the predicted $\mathrm{H} \mathrm{I}$ emissionline spectrum is changed with respect to the C07.02. Input parameters for the simulations are chosen to match the standard conditions in the BLR (Ferland 2006; Korista \& Goad 2000, 2004). Assuming solar chemical abundances and a constant hydrogen density, and using the code's AGN template for the incident continuum shape (a continuum similar to the typical radioquiet active galaxies), we compute an emission-line spectrum for the coordinate pair of hydrogen gas density $n_{\mathrm{H}}\left[\mathrm{cm}^{-3}\right]$ and hydrogen-ionizing photon flux $\Phi_{\mathrm{H}}\left[\mathrm{cm}^{-2} \mathrm{~s}^{-1}\right]$.

The grid dimensions span six orders of magnitude in each direction and, have an origin in $\log n_{\mathrm{H}}=8, \log \Phi_{\mathrm{H}}=17$ and a step of 0.2 dex increments, produced a total of 961 simulations per grid. The column density $N_{\mathrm{H}}\left[\mathrm{cm}^{-2}\right]$ is kept constant in producing the grid of simulations. Many authors claim that
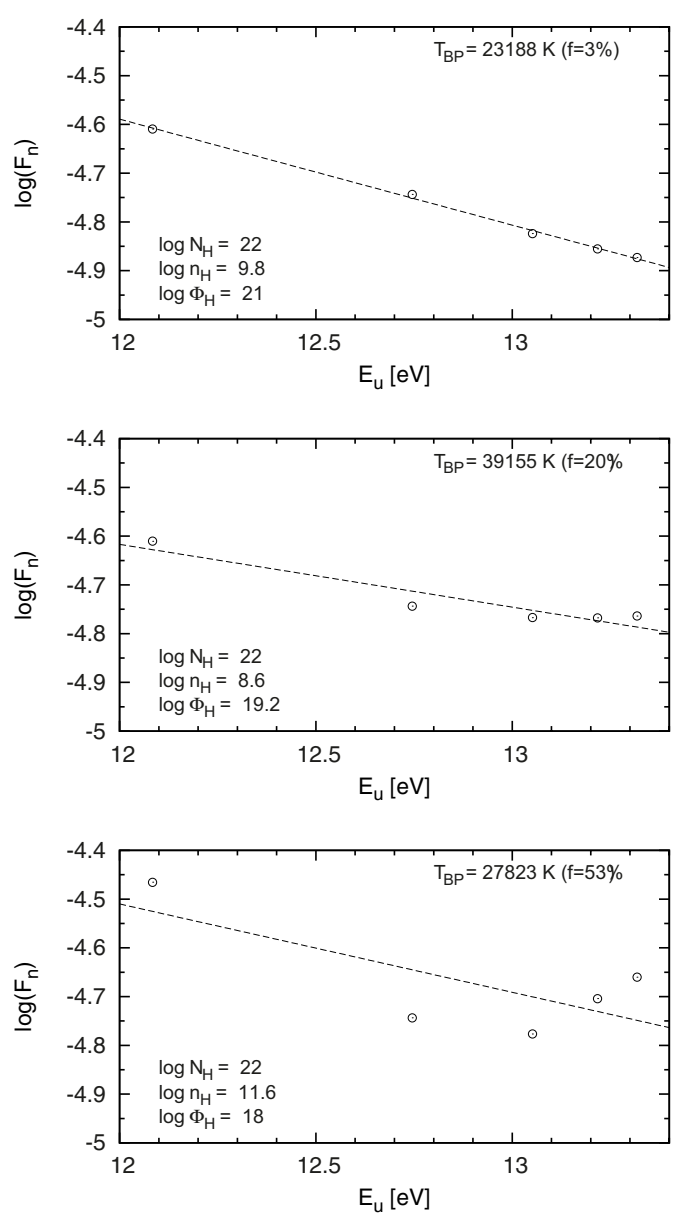

Fig. 2. Different examples of the BP applied to the Balmer lines obtained with the CLOUDY models. The intensities $F_{\mathrm{n}}$ are calculated using the Balmer lines normalized to the $\mathrm{H} \beta$ flux. In the upper right corner, the BP temperature $T_{\mathrm{BP}}$ and the error in the BP fit $f$ (in percentage) are given. In the bottom left corner, the column density $N_{\mathrm{H}}$, the hydrogen density $n_{\mathrm{H}}$, and the input ionizing flux $\Phi_{\mathrm{H}}$ are given.

the most probable value of the column density in the BLR is $N_{\mathrm{H}}=10^{23} \mathrm{~cm}^{-2}$ (Dumont et al. 1998; Korista \& Goad 2000, 2004). Since the column density defines the optical thickness of the region, here we produce three grids of models changing the column density between $N_{\mathrm{H}}=\left[10^{21}, 10^{22}, 10^{23}\right] \mathrm{cm}^{-2}$. Therefore, the total number of simulations is 2883 .

The output of the code includes all lines ${ }^{3}$ formed in the emitting region, with a given chemical composition, hydrogen density, and hydrogen-ionizing photon flux. We consider in our analysis the strongest hydrogen Balmer lines ( $\mathrm{H} \alpha$ to $\mathrm{H} \varepsilon$ ).

\subsubsection{Modeled line ratios}

For every grid of CLOUDY models, we analyze the emission line fluxes by applying the BP method to the Balmer lines. In particular, using Eq. (3), we estimate the parameter $A$, from which we then calculate the BP temperature (hereinafter denoted as $T_{\mathrm{BP}}$ ) of the emitting region and the error associated with the best fit in the BP, which we denote as $f$.

A few examples of the BP applied to the simulated Balmer lines for a column density of $N_{\mathrm{H}}=10^{22} \mathrm{~cm}^{-2}$ are presented in Fig. 2. In most cases, a satisfactory fit of Eq. (3) is not obtained

${ }^{3}$ By default, the code gives line fluxes normalized to the $\mathrm{H} \beta$ flux. Since it has no influence on the BP analysis, we have used the normalized values. 
D. Ilić et al.: The analysis of the broad hydrogen Balmer line ratios in the BLR of AGNs
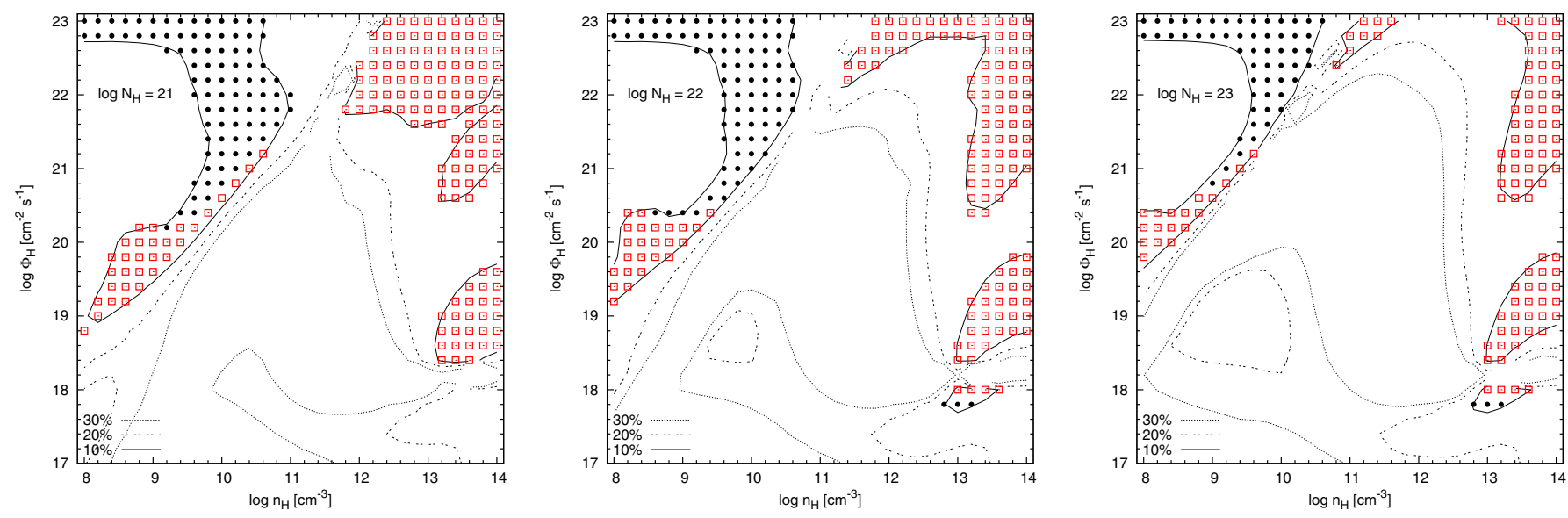

Fig. 3. Contour plots for the error in the BP fit $f$ in the ionizing flux and hydrogen density plane for different column densities $N_{\mathrm{H}} \in$ $\left[10^{21}-10^{23}\right] \mathrm{cm}^{-2}$. The curves show the regions where $f$ is smaller than $10 \%, 20 \%$, and $30 \%$, while open squares represent the simulations for which $f \leq 10 \%$, and full circles are the simulations for which $f \leq 10 \%$ and $T_{\mathrm{BP}}<30000 \mathrm{~K}$.

and $f$ has quite large values. This is more noticeable in the plots of $f$ in the hydrogen density and ionizing flux plane for all three grids of models, illustrated in Fig. 3. It can be seen in Fig. 3 that the input parameters $\Phi_{\mathrm{H}}$ and $n_{\mathrm{H}}$ for which $f$ was smaller than $10 \%$ are well-constrained (open squares in Fig. 3), and cover a similar range for different column densities. The realistic models for which $T_{\mathrm{BP}}<30000 \mathrm{~K}$ (denoted with full circles in Fig. 3) have a very high ionization parameter (low density and high input ionization flux), except for a couple of cases with high densities appearing at higher column densities.

\subsection{Theoretical line ratios}

We also consider theoretical recombination Balmer lines calculated by Hummer \& Storey (1987, 1992); Storey \& Hummer $(1988,1995 a)$. We wish to analyze the hydrogen Balmer lines obtained only using the recombination theory, and compare them to the line ratios obtained by the CLOUDY code, which, on the other hand, treats simultaneously millions of lines and physical processes, so that the behavior of the hydrogen lines might be hidden.

Storey \& Hummer (1995a) calculated the line emissivities, together with other line transition parameters for hydrogenic atoms, assuming both Case A and Case B recombination, and including full collisional effects for a considerably large range of temperature, density, and principal quantum numbers. Their calculated data are given in tables available in an online catalogue ${ }^{4}$ (see Storey \& Hummer 1995a,b, for details).

Here we use their theoretical hydrogen Balmer lines $(\mathrm{H} \alpha$ to $\mathrm{H} \varepsilon$ ), calculated for the Case $\mathrm{B}$ recombination with the density $\log n_{\mathrm{e}}$ in the range (8.2-14, 0.2 dex increment), and the electron temperature lying within the range from $1000 \mathrm{~K}$ to $30000 \mathrm{~K}$ (in steps of $1000 \mathrm{~K}$ ). Online data provide line emissivities within this range, but only for a small number of values of density and temperature (Storey \& Hummer 1995a), for others we interpolated line emissivities using the interpolation code provided by Storey \& Hummer (1995a). For the BP method, we use the ratios of Balmer lines, which we can directly derive from line emissivities.

We plot the error in the BP fit $f$ for theoretical Balmer line ratios in the electron temperature and electron density plane

\footnotetext{
${ }^{4}$ http://vizier.cfa.harvard.edu/viz-bin/VizieR? - source $=V I / 64$
}

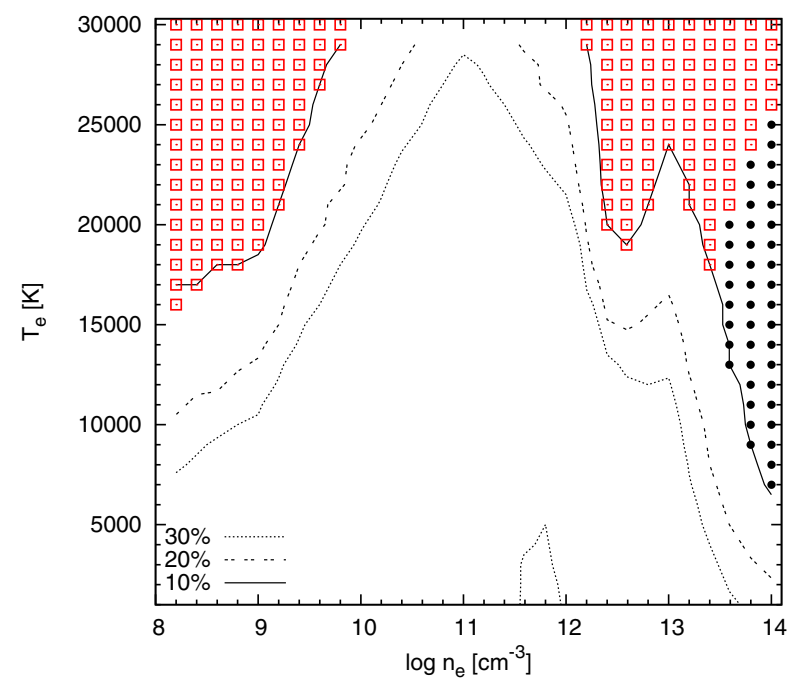

Fig. 4. Contour plot for the error in the BP fit $f$ in the electron temperature versus (vs.) electron density plane for theoretical Balmer line ratios. The curves show the regions where $f$ is smaller than $10 \%, 20 \%$, and $30 \%$, while points have the same meaning as in Fig. 3.

(Fig. 4). Here we do not have the input ionizing flux, which is used as an input parameter for modeled line ratios. However, we can say that basically the electron temperature gives some indications about the input ionizing flux. For the theoretical line ratios, we find that the $f \leq 10 \%$ area also covers a constrained area of parameter space (open squares in Fig. 4), which resembles that inferred from the simulated line ratios. Here the cases with $T_{\mathrm{BP}}<30000 \mathrm{~K}$ (denoted with full circles in Fig. 4) appear for high densities $\sim 10^{13-14} \mathrm{~cm}^{-3}$.

The BP temperature as a function of the electron temperature is plotted in Fig. 5, for cases when the BP fit error is smaller than $10 \%$. There is clearly a relation between these two temperatures, especially for the electron density of $10^{14} \mathrm{~cm}^{-3}$ (open circles in Fig. 5). The BP temperature, which represents the excitation temperature, is higher than the electron temperature. The linear best-fitting gives the following relation $T_{\mathrm{BP}}=3133+1.06 \times T_{\mathrm{e}}$.

The Balmer decrement when $f \leq 10 \%$ follows the behavior of the Boltzmann plot (solid line in Fig. 6). For these cases in 


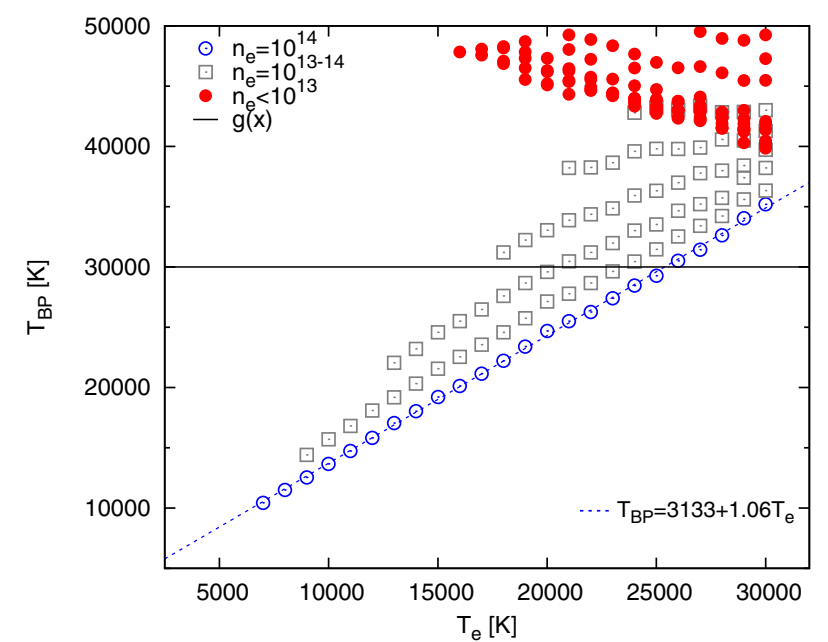

Fig. 5. Boltzmann-plot temperature plotted against the electron temperature for the case when $f \leq 10 \%$. Open circles are for the electron density of $10^{14} \mathrm{~cm}^{-3}$, open squares for $10^{13} \leq n_{\mathrm{e}}<10^{14} \mathrm{~cm}^{-3}$, and full circles for $n_{\mathrm{e}}<10^{13} \mathrm{~cm}^{-3}$. The linear best-fitting of the cases with $n_{\mathrm{e}}=10^{14} \mathrm{~cm}^{-3}$ is given with the dashed line. The solid line corresponds to $T_{\mathrm{BP}}=30000 \mathrm{~K}$.

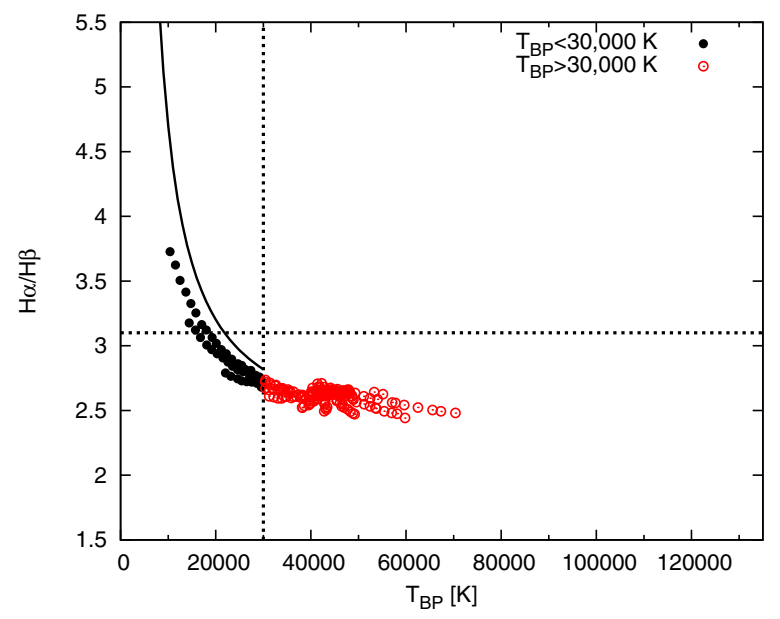

Fig. 6. $\mathrm{H} \alpha / \mathrm{H} \beta$ ratio plotted against the BP temperature for $f \leq 10 \%$, where full circles denote $T_{\mathrm{BP}}<30000 \mathrm{~K}$ and open circles $T_{\mathrm{BP}}>$ $30000 \mathrm{~K}$, and are separated with the vertical dashed line. The horizontal dashed line represents $\mathrm{H} \alpha / \mathrm{H} \beta=3.1$. The solid line gives the line ratio calculated using Eq. (1).

Fig. 6, the $\mathrm{H} \alpha / \mathrm{H} \beta$ ratio is shown against the BP temperature. The solid line represents the Balmer decrement calculated for different temperatures using Eq. (1), and it is shown only for temperatures lower than $30000 \mathrm{~K}$. The average $\mathrm{H} \alpha / \mathrm{H} \beta$ is 2.96 for cases when $T_{\mathrm{BP}}<30000 \mathrm{~K}$ (full circles in Fig. 6).

\section{Observed line ratios}

We apply the BP method to the observed data. We consider measurements performed on a sample of 90 broad-lineemitting AGNs, taken from the SDSS spectral database. For this sample, the Balmer line parameters were accurately estimated by considering the complexity of BEL (see La Mura et al. 2007, for details). We repeat here that the line fluxes are corrected for the Galactic extinction, as well as the host galaxy contribution,

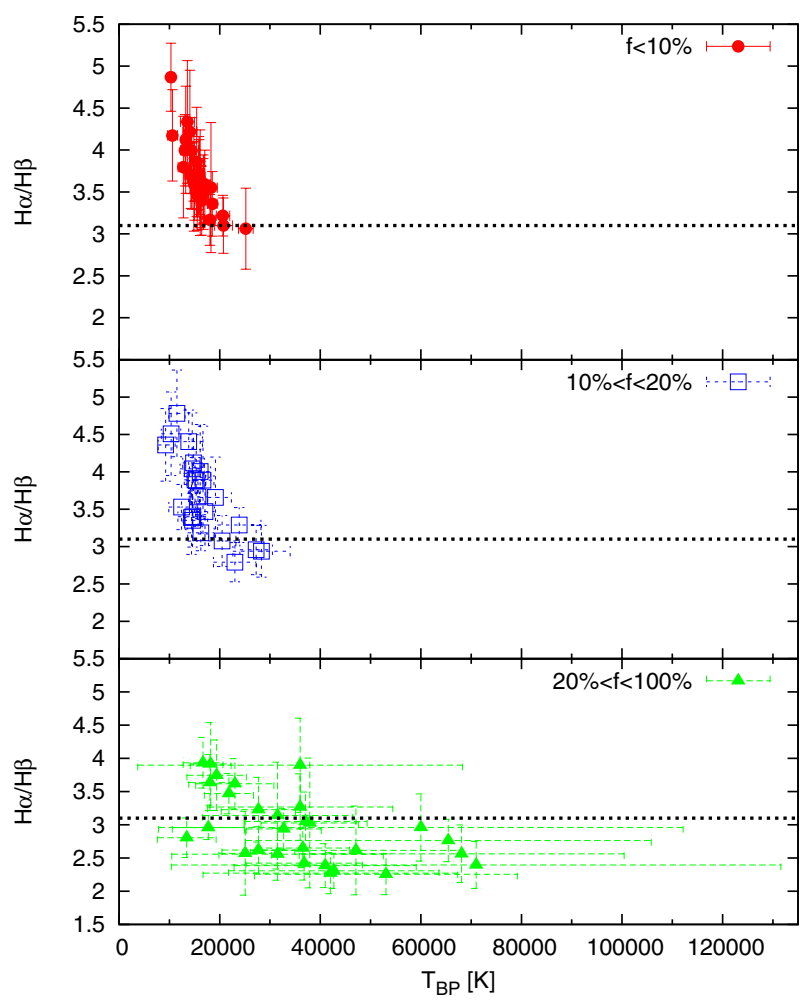

Fig. 7. $\mathrm{H} \alpha / \mathrm{H} \beta$ ratio vs. the BP temperature for the sample of SDSS galaxies for objects that have the BP fit error $f \leq 10 \%$ (upper panel, full circles), objects with $10 \%<f \leq 20 \%$ (middle panel, open squares), and with $20 \%<f \leq 100 \%$ (bottom panel, full triangles) are given. The dashed line represents $\mathrm{H} \alpha / \mathrm{H} \beta=3.1$.

which to some extent corrects for the internal dust extinction, but not the dust effects within the BLR. The BP method, applied to the broad component of Balmer lines of this sample (Fig. 1), produces reasonably good fit uncertainties, with $f \leq 10 \%$, in approximately $35 \%$ of cases. The BP temperatures are in the range $T_{\mathrm{BP}}=10300-25200 \mathrm{~K}$. We consider the cases in which $f \leq 10 \%$ to calculate $T_{\mathrm{e}}$ assuming that the electron density is $n_{\mathrm{e}}=10^{14} \mathrm{~cm}^{-3}$ and using the linear relation from Fig. 5. We obtain the electron temperature in the range $T_{\mathrm{e}}=7350-22250 \mathrm{~K}$. The obtained values for both temperatures are in good agreement with the values considered to be typical for the BLR plasma.

We plot the $\mathrm{H} \alpha / \mathrm{H} \beta$ ratio against the $\mathrm{BP}$ temperature for this sample of 90 SDSS galaxies (Fig. 7) denoting differently objects with different $f$. Objects for which the BP method is applicable (full circles, upper panel Fig. 7) tend to have higher $\mathrm{H} \alpha / \mathrm{H} \beta$ ratios (average value is 3.68), in contrast to objects with large fitting errors $f$ (full triangles, bottom panel 7), which mostly have low Balmer decrements, below 3.1 (dashed line, Fig. 7).

One indication of the Balmer lines optical depths can be seen if we compare their normalized-line profiles. Some examples of the line-profiles comparison from the SDSS sample of galaxies for which $f \leq 10 \%$ are plotted in Fig. 8. The $\mathrm{H} \alpha$, $\mathrm{H} \beta$, and $\mathrm{H} \gamma$ line profiles (only the broad component) are compared after being smoothed according to their spectral signalto-noise $(\mathrm{S} / \mathrm{N})$ ratio and normalized to unity. From the sample of 90 objects, objects with small BP errors $f$ contain $\mathrm{H} \alpha, \mathrm{H} \beta$, and $\mathrm{H} \gamma$ lines with similar line profiles (Fig. 8), implying that these lines may be optically thin. One should keep in mind the relatively low resolution of the SDSS, which is especially evident in the case of lines with small full widths at half maximum, whose line profiles are significantly affected by this. 
D. Ilić et al.: The analysis of the broad hydrogen Balmer line ratios in the BLR of AGNs
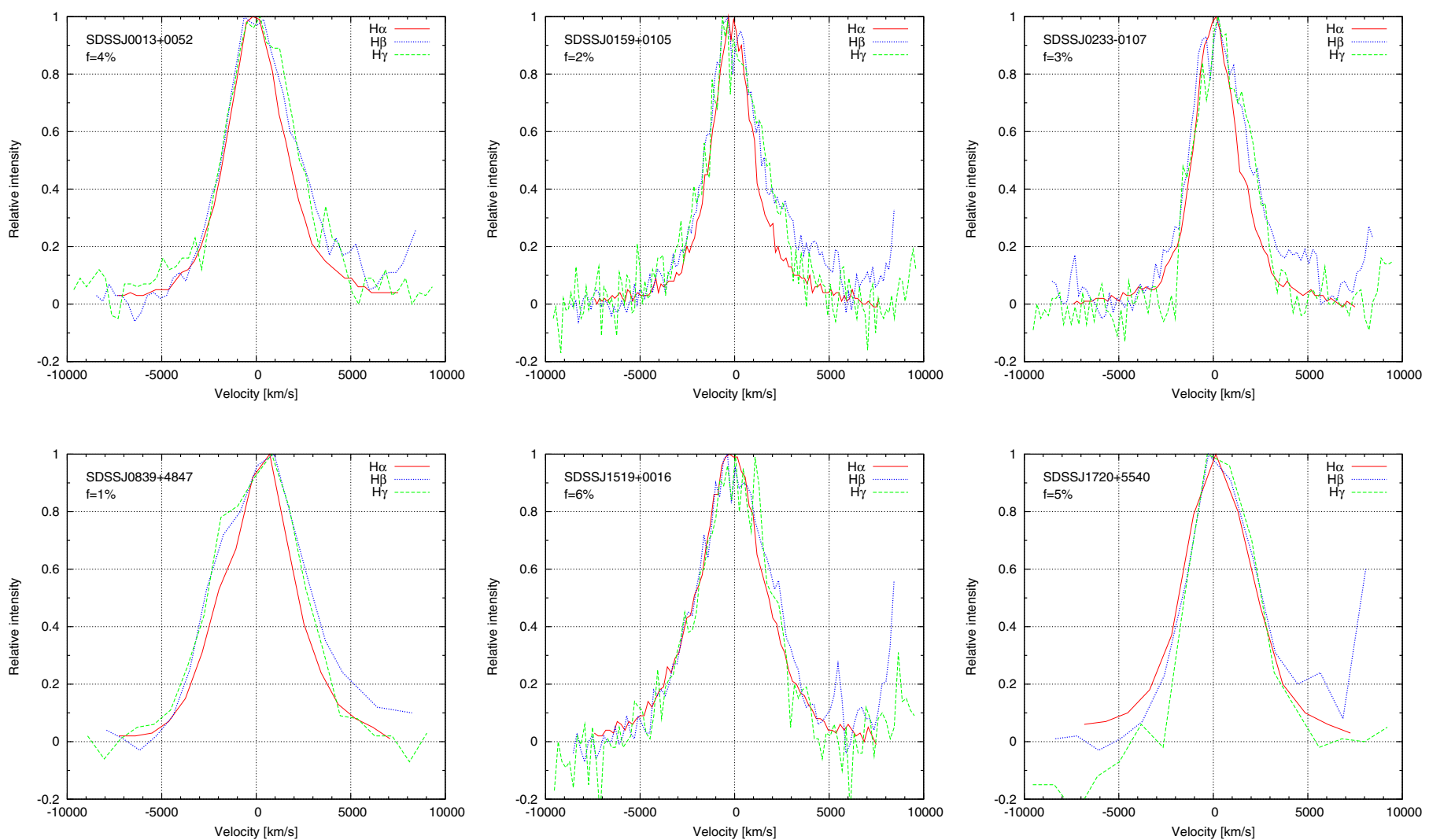

Fig. 8. Some examples of the line-profiles comparison for the SDSS galaxies for which the BP fit error is smaller than $10 \%$. The H $\alpha$ (solid line), $\mathrm{H} \beta$ (dotted line), and $\mathrm{H} \gamma$ (dashed line) broad components are shown. The name of the SDSS galaxy and the BP error $f$ are given. Lines are smoothed according to the spectral $\mathrm{S} / \mathrm{N}$ ratio, normalized to unity, and converted to the velocity scale.

\section{Discussion}

According to the simulation results, we have been able to constrain a parameter space, for different column densities $N_{\mathrm{H}}=$ $10^{21}-10^{23} \mathrm{~cm}^{-2}$, where the BP produces reasonable results with small fit uncertainties $(f \leq 10 \%)$. Here the cases with $T_{\mathrm{BP}}<$ $30000 \mathrm{~K}$ (denoted with full circles, Fig. 3), which represent fewer than $9 \%$ of the total number of simulations, mostly fall within an area with higher ionizing fluxes and lower hydrogen densities, which are indicative of high ionization parameters. These situations are extreme ones, thus the most likely regions of the BP should be those of higher density, as seen in the case of higher column densities (Fig. 3).

A similar trend is seen for the theoretical line ratios (Fig. 4). Here the parameter range where the BP method is most applicable is constrained and higher densities are preferred, namely about $\sim 10^{14} \mathrm{~cm}^{-3}$ (Fig. 5). In these situations, even if photoionization were to act as the main heating source for the BLR, a contribution of the collisional processes may become relevant (especially for such high densities) and significantly affect the distribution of the excited energy configuration of the ions. The BP method reveals the occurrence of these circumstances and provides an estimate of the excitation temperature, which is basically described by the Boltzmann equation of the hydrogen energy levels.

In the sample of 90 broad-line-emitting sources from SDSS, there are more than $30 \%$ cases in which the BP can be applied that give reasonably good fit uncertainties $(f \leq 10 \%)$, which we denote as the "BP objects". From the SDSS observations, we can see that the BP objects have a larger Balmer decrement (Fig. 7), with an average value of $\mathrm{H} \alpha / \mathrm{H} \beta=3.68$. This value is larger than the average value of 3.06 of the broad-line Balmer decrement of Dong et al. (2008) obtained for blue type 1 AGNs. Our measured Balmer decrement may indicate two situations: (i) either there is a relatively strong contribution from collisional processes, i.e. we have high density plasma in the BLR, as also supported by the recombination theory and modeled line ratios, and suggested in some earlier works (van Groningen 1987; Sivron \& Tsuruta 1993; Brotherton et al. 1994), (ii) or that such a high $\mathrm{H} \alpha / \mathrm{H} \beta$ ratio is caused by intrinsic dust extinction effects (see discussion in Sect. 2.1). On the other hand, the measured Balmer decrements and the temperature parameter $A$ (see Eq. (1)) of the SDSS objects that follow the BP are not necessarily indicative of high continuum luminosities (see Fig. 11 in La Mura et al. 2007). If we put this in the context of the modeling and theory findings, we can say that these objects then tend to have a higher gas density in the BLR.

We now discuss the possible mechanism of line production in such BLR plasma, especially the requirements to have optically thin Balmer lines. We start from the basic assumption that we have relatively high opacities in the Lyman line series, while the Balmer line series is optically thin. In such a case, the $\operatorname{Ly} \alpha$ line is scattered, while the $\operatorname{Ly} \beta$ is almost completely suppressed. Therefore, atoms are constantly excited to the energy level $n=2$. Since the energy gap between the $n=2$ and $n=3$ level is $1.9 \mathrm{eV}$, corresponding to an electron temperature of approximately $23000 \mathrm{~K}$, collisional excitation occur not only to the $n=3$ level, but also to $n=4$. The lifetimes of the levels $n=3,4$ are shorter than those for $n=2$, so we observe that both $\mathrm{H} \alpha$ and $\mathrm{H} \beta$ lines are produced. Even if the lifetime of the $n=2$ level is also short, this level is constantly populated by Ly $\alpha$ scattering. Therefore, the population of the energy levels 
in the Balmer line series may be described by the Boltzmann distribution, which is defined by the excitation temperature.

The line profiles of the Balmer lines in the observed "BP objects" are similar (Fig. 8), indicating that these lines are close to being optically thin. Therefore, we can state that the objects that follow the BP, the so-called "BP objects" have broad-line hydrogen Balmer lines that may originate from the BLR clouds of high density $\sim 10^{14} \mathrm{~cm}^{-3}$, when the broad lines are not influenced by the intrinsic dust effects. Moreover, these so-called "BP objects" tend to have broader BEL indicating that the regions where the BP fit works should have higher emitter velocities and be closer to the central engine (see Fig. 9 in La Mura et al. 2007).

Finally, we note that the BLR plasma cannot be generally considered to be in the equilibrium regime for which the Boltzmann distribution can be used, since the density of emitters is probably non-uniform across the region. The density can be expressed as (Osterbrock \& Ferland 2006): $N_{m} \sim$ $b_{m}\left(T, n_{\mathrm{e}}\right) g_{m} \exp \left(-E_{m n} / k T\right)$ where $b_{m}\left(T, n_{\mathrm{e}}\right)$ represents the deviation from thermodynamical equilibrium. In general, photoionization models predict that $b\left(T, n_{\mathrm{e}}\right) \neq 1$, but, taking into account the ratio of emission lines belonging to the same series, the deviation from thermodynamical equilibrium might be overtaken if the condition $b_{i}\left(T, n_{\mathrm{e}}\right) / b_{j}\left(T, n_{\mathrm{e}}\right) \approx 1$ is met, which should be possible at least for higher energy levels (Osterbrock \& Ferland 2006).

\section{Conclusions}

We have presented a study of the Balmer line ratios of AGNs and their usage as a tool to investigate the physical properties of the broad line region of AGNs. Our main results are based on the analysis of the broad hydrogen Balmer lines ( $\mathrm{H} \alpha$ to $\mathrm{H} \varepsilon$ ). Lineratios predictions have been obtained from the photoionization models generated by the spectral synthesis code CLOUDY (version C08.00), calculated using the recombination theory for hydrogenic ions, and measured from the sample of observed spectra of 90 broad-line AGNs from SDSS. The Boltzmann plot was applied to all samples and the $T_{\mathrm{BP}}$ obtained. The properties of spectra with the BP error $f \leq 10 \%$ and plasma conditions for their formation have been explored. From our analysis, we have arrived at these conclusions:

i) From the CLOUDY photoionization models, we have found that for a limited space of physical parameter $\left(n_{\mathrm{H}}, \Phi_{\mathrm{H}}\right)$ the physical processes in plasma are such that the Balmer lines follow the BP. This is the case for either very high ionization parameter (low density and high input ionization flux) or for high densities at higher column densities.

ii) The recombination theory for hydrogenic ions predicts that the BP may be applicable to higher densities $\sim 10^{14} \mathrm{~cm}^{-3}$.

iii) From the analysis of the sample of broad line AGNs from the SDSS database, it follows that the BP can be applied in $\approx 35 \%$ of cases, and the obtained BLR temperatures are in the range $T_{\mathrm{BP}}=10300-25200 \mathrm{~K}$.

There is a fraction of objects that follow the BP ("BP objects"), indicating that in their BLR the physical conditions ensure that the hydrogen populations in their upper energy states $(n \geq 3)$ follow the Saha-Boltzmann distribution. These objects are thus interesting and should be investigated in greater detail (e.g. to determine their UV or X-ray spectral characteristics, or their connection to other AGN types), a study that will be the subject of future work.
Acknowledgements. D.I. would like to thank the Department of Physics and Astronomy of the University of Padova, Italy for their hospitality. We also, thank the anonymous referee for useful comments. This work was supported by the Ministry of Education and Science of the Republic of Serbia through the project Astrophysical Spectroscopy of Extragalactic Objects (\#176001). Funding for the SDSS and SDSS-II has been provided by the Alfred P. Sloan Foundation, the Participating Institutions, the National Science Foundation, the US Department of Energy, the National Aeronautics and Space Administration, the Japanese Monbukagakusho, the Max Planck Society, and the Higher Education Funding Council for England. The SDSS is managed by the Astrophysical Research Consortium (ARC) for the Participating Institutions. The SDSS Web Site is http: //www. sdss.org/. This research has made use of NASA's Astrophysics Data System.

\section{References}

Baldwin, J. A. 1997, in Emission Lines in Active Galaxies: New Methods and Techniques, IAU Colloq. 159, eds. B. M. Peterson, F. Cheng, \& A. S. Wilson (San Francisco: ASP), ASP Conf. Ser., 113, 80

Baldwin, J. A., Ferland, G. J., Korista, K. T., \& Verner, D. A. 1995, ApJ, 455, L119

Baldwin, J. A., Ferland, G. J., Korista, K. T., et al. 1996, ApJ, 461, 664

Bentz, M. C., Walsh, J. L., Barth, A. J., et al. 2010, ApJ, 716, 993

Bon, E., Popović, L. Č., Gavrilović, N., Mura, G. La, \& Mediavilla, E. 2009, MNRAS, 400, 924

Brotherton, M. S., Wills, B. J., Steidel, R., \& Sargent, W. L. W. 1994, ApJ, 423, 131

Canfield, R. C., \& Puetter, R. C. 1981, ApJ, 243, 390

Collin-Souffrin, S., \& Lasota, J.-P. 1988, PASP, 100, 1041

Collin-Souffrin, S., Dumont, S., \& Tully, J. 1982, A\&A, 106, 362

Crenshaw, D. M., Peterson, B. M., \& Wagner, R. M. 1988, AJ, 96, 1208

Davidson, K., \& Netzer, H. 1979, RvMP, 51, 715

Dumont, A.-M., Collin-Souffrin, S., \& Nazarova, L. 1998, A\&A, 331, 11

Dong, X.-B., Zhou, H.-Y., Wang, T.-G., et al. 2005, ApJ, 620, 629

Dong, X., Wang, T., Wang, J., et al. 2008, MNRAS, 383, 581

Ferland, G. J. 1999, in Quasars and Cosmology, eds. G. Ferland, \& J. Baldwin, ASP Conf. Ser., 162, 147

Ferland, G. J. 2003, ARA\&A, 41, 517

Ferland, G. J. 2006, Hazy, A Brief Introduction to Cloudy 06.02, University of Kentucky Internal Report

Ferland, G. J., Korista, K. T., Verner, D. A., et al. 1998, PASP, 110, 761

Ferland, G. J., \& Savin, D. W. 2001, Spectroscopic Challenges of Photoionized Plasmas, ASP Conf. Ser., 247 (San Francisco: ASP)

Gaskell, C. M. 2009, New Astron. Rev., 53, 140

Gaskell, C. M., \& Ferland, G. J. 1984, PASP, 96, 393

Goodrich, R. W. 1995, ApJ, 440, 141

Griem, H. R. 1997, Principles of plasma spectroscopy (Cambridge: Cambridge University Press)

van Groningen, E. 1987, A\&A, 186, 103

Halpern, J. P., \& Steiner, J. E. 1983, ApJ, 269, L37

Hummer, D. G., \& Storey, P. J. 1987, MNRAS, 224, 801

Hummer, D. G., \& Storey, P. J. 1992, MNRAS, 254, 277

Ilić, D., Popović, L. Č., Bon, E., Mediavilla, E., \& Chavushyan, V. H. 2006, MNRAS, 371, 1610

Ilić, D., Kovačević, J., \& Popović, L. Č. 2009, New Astron. Rev., 53, 149

Jin, C., Ward, M., Done, C., \& Gelbord, J. 2012, MNRAS, 420, 1825

Konjević, N. 1999, PhR, 316, 339

Korista, K. T., \& Goad, M. R. 2000, ApJ, 536, 284

Korista, K. T., \& Goad, M. R. 2004, ApJ, 606, 749

Kovačević, J., Popović, L. Č., \& Dimitrijević, M. S. 2010, ApJS, 189, 15

Krolik, J. H. 1999, Active Galactic Nuclei: From the Central Black Hole to the Galactic Environment (Princeton, New Yersey: Princeton University Press)

Kwan, J., \& Krolik, J. 1981, ApJ, 233, L91

Kwan, J., \& Krolik, J. 1981, ApJ, 250, 478

La Mura, G., Popović, L. Č., Ciroi, S., Rafanelli, P., \& Ilić, D. 2007, ApJ, 671, 104

La Mura, G., Di Mille, F., Ciroi, S., Popović, L. Č., \& Rafanelli, P. 2009, ApJ, 693, 1437

Laor, A. 2006, ApJ, 643, 112

Laor, A., Jannuzi, B. T., Green, R. F., \& Boroson, T. A. 1997, ApJ, 489, 656

Leighly, K. M., \& Casebeer, D. 2007, The Central Engine of Active Galactic Nuclei, 373, 365

Marziani, P., Sulentic, J. W., Zwitter, T., Dultzin-Hacyan, D., \& Calvani, M. 2001, ApJ, 558, 553

Marziani, P., Sulentic, J. W., Negrete, C. A., et al. 2010, MNRAS, 409, 1033

Mathews, W. G., Blumenthal, G. R., \& Grandi, S. A. 1980, ApJ, 235, 971 
D. Ilić et al.: The analysis of the broad hydrogen Balmer line ratios in the BLR of AGNs

McGill, K. L., Woo, J.-H., Treu, T., \& Malkan, M. 2008, ApJ, 673, 703

Negrete, C. A., Dultzin, D., Marziani, P., \& Sulentic, J. W. 2011 [arXiv: 1107.3188]

Netzer, H. 1975, MNRAS, 171, 395

Netzer, H., Brotherton, M. S., Wills, B. J., et al. 1995, ApJ, 448, 27

Osterbrock, D. E. 1977, ApJ, 215, 733

Osterbrock, D. E. 1978, PNAS, 75, 540

Osterbrock, D. E. 1981, ApJ, 249, 462

Osterbrock, D. E. 1984, QJRAS, 25, 1

Osterbrock, D. E., \& Ferland, G. J. 2006, Astrophysics of Gaseous Nebulae and Active Galactic Nuclei, 2nd edn. (Sausalito, California: University Science Books)

Peterson, B. M. 1994, in Reverberation Mapping of the Broad-Line Region in Active Galactic Nuclei, 69, 1

Popović, L. Č. 2003, ApJ, 599, 140

Popović, L. Č. 2006a, ApJ, 650, 1217 (an Erratum)

Popović, L. C. 2006b, SerAJ, 173, 1

Popović, L. Č., Mediavilla, E. G., Kubičela, A., \& Jovanović, P. 2002, A\&A, 390,473
Popović, L. Č., Mediavilla, E. G., Bon, E., \& Ilić, D. 2004, A\&A, 423, 909 Popović, L. Č., Shapovalova, A. I., Chavushyan, V. H., et al. 2008, PASJ, 61, 1 Popović, L. C., Smirnova, A. A., Kovačević, J., Moiseev, A. V., \& Afanasiev, V. L. 2009, AJ, 137, 3548

Popović, L. Č., Shapovalova, A. I., Ilić, D., et al. 2011, A\&A, 528, A130

Rafanelli, P. 1985, A\&A, 146, 17

Rees, M., Netzer, H., \& Ferland, G. J. 1989, ApJ, 347, 640

Sivron, R., \& Tsuruta, S. 1993, ApJ, 402, 420

Snedden, S. A., \& Gaskell, C. M. 2007, ApJ, 669, 126

Shapovalova, A. I., Doroshenko, V. T., Bochkarev, N. G., et al. 2004, A\&A, 422, 925

Shapovalova, A. I., Popović, L. Č., Collin, S., et al. 2008, A\&A, 486, 99

Shapovalova, A. I., Popović, L. C.., Burenkov, A. N., et al. 2010, A\&A, 509, A106

Storey, P. J., \& Hummer, D. G. 1988, MNRAS, 231, 1139

Storey, P. J., \& Hummer, D. G. 1995a, MNRAS, 272, 41

Storey, P. J., \& Hummer, D. G. 1995b, VizieR Online Data Catalog, VI/064

Sulentic, J. W., Marziani, P., \& Dultzin-Hacyan, D. 2000, ARA\&A, 38, 521

Sulentic, J. W., Marziani, P., \& Zamfir, S. 2009, New Astron. Rev., 53, 198 